\title{
Dietary intake of animal and plant proteins and risk of all cause and cause-specific mortality: The Epic-Italy cohort
}

\author{
Luigi Fontana ${ }^{\mathrm{a}, \mathrm{b}, \mathrm{c}, 1, *}$, Sabina Sieri ${ }^{\mathrm{d}, 1}$, Fulvio Ricceri ${ }^{\mathrm{e}, \mathrm{f}}$, Claudia Agnoli ${ }^{\mathrm{d}}$, Valeria Pala ${ }^{\mathrm{d}}$, \\ Giovanna Masala ${ }^{\mathrm{g}}$, Calogero Saieva $^{\mathrm{g}}$, Alberto Catalano ${ }^{\mathrm{f}}$, Alessandra Macciotta $^{\mathrm{f}}$, Rosario Tumino ${ }^{\mathrm{h}}$, \\ Salvatore Panico ${ }^{\mathrm{i}}$, Maria Santucci De Magistris ${ }^{\mathrm{i}}$ and Vittorio Krogh ${ }^{\mathrm{d}}$ \\ ${ }^{a}$ Charles Perkins Centre, Faculty of Medicine and Health, University of Sydney, Sydney, NSW, Australia \\ ${ }^{\mathrm{b}}$ Department of Endocrinology, Royal Prince Alfred Hospital, Sydney, NSW, Australia \\ ${ }^{\mathrm{c}}$ Department of Clinical and Experimental Sciences, Brescia University School of Medicine, Brescia, Italy \\ ${ }^{\mathrm{d}}$ Epidemiology and Prevention Unit, Fondazione IRCCS Istituto Nazionale dei Tumori, Milan, Italy \\ ${ }^{\mathrm{e}}$ Epidemiology Unit, Regional Health Services ASL TO3, Grugliasco, Italy \\ ${ }^{\mathrm{f}}$ Department of Clinical and Biological Sciences, University of Turin, Turin, Italy \\ ${ }^{\mathrm{g}}$ Institute for Cancer Research, Prevention and Clinical Network (ISPRO), Florence, Italy \\ ${ }^{\mathrm{h}}$ Cancer Registry and Histopathology Department, Civic-M. P. Arezzo Hospital, Azienda Sanitaria Provinciale, \\ Ragusa, Italy

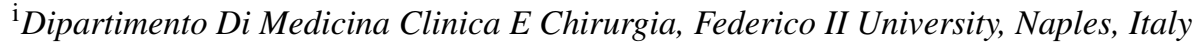

Received 22 November 2021

Accepted 21 January 2022

Pre-press 11 February 2022

Published 13 April 2022

\begin{abstract}
.
\footnotetext{
${ }^{1}$ Authors have equally contributed to this research.

*Corresponding author: Professor Luigi Fontana, MD, PhD, FRACP, Professor of Medicine and Nutrition, Leonard P Ullman Chair in Translational Metabolic Health, Director, Healthy Longevity Research and Clinical Program, Charles Perkins Cen-
}

BACKGROUND: To examine the associations of animal and plant protein intake with all-cause, cardiovascular and cancer mortality risk in middle-aged Italian men and women with substantially lower animal protein intake than North Americans. METHODS AND RESULTS: Food consumption was assessed by validated Epic semiquantitative FFQs. Multivariable Cox models stratified by center, age, and sex, and adjusted for confounders, estimated associations of animal and plant protein consumption with mortality for all causes, cardiovascular disease, and cancer. After a median follow-up of 15.2 years, 2,449 deaths were identified in 45,009 participants. No significant association between intake of total, animal or plant protein and mortality was found in the fully adjusted models. Substitution of plant protein for animal protein was inversely associated with cardiovascular mortality (HR, 0.47; 95\% CI, 0.24-0.92) only in people with at least 1 unhealthy lifestyle risk factor and poor adherence to a Mediterranean diet. Participants in the highest quintile group of animal protein intake had higher glucose, total and LDL cholesterol levels than those in the lowest quintile. In contrast, higher plant protein intake was negatively associated with fasting insulin and cholesterol, despite higher BMI, physical inactivity and starch consumption.

CONCLUSIONS: Replacing plant protein for animal protein was associated with lower cardiovascular mortality among individuals with unhealthy lifestyle risk factors. High animal but not plant protein intake is associated with impaired fasting

tre, Central Clinical School, Faculty of Medicine and Health, Level 5 West, D17 - Education and Research Hub, The Charles Perkins Centre, The University of Sydney, NSW 2006, Australia. Tel.: +61 28627 7499; +61 40879 0318; E-mail: luigi.fontana@sydney.edu.au.; http://sydney.edu.au/perkins. 
glucose and hypercholesterolemia, despite lower calorie and carbohydrate intake, suggesting that protein source plays crucial roles in modulating cardiometabolic health independently of body weight.

Keywords: Protein intake, animal protein, vegetable protein, mortality, cardiovascular disease, lifestyle risk factors, Mediterranean diet, cholesterol

\section{Introduction}

An overwhelming body of data indicate that lower intake of dietary protein, methionine, tryptophan and branched chain amino acids, independently of energy intake, improve health and extend lifespan in multiple model organisms including rodents [1-4]. Results from human epidemiological studies on the association between total and animal protein consumption and mortality are still contentious, with some reporting an inverse association, but many no or even a positive relationship [5, 6]. In contrast, most studies show a protective effect of plant proteins that typically have lower content of methionine, tryptophan and branched chain amino acids [5-9].

Several residual or unmeasured dietary and metabolic confounding factors may explain these discrepancies. For example, animal protein consumption (and associated iron and saturated fat content) is considerably higher in the large North American study populations compared to those living in Southern Europe countries like Italy [7]. Moreover, as it has been shown for the BMI-mortality relationship, the protein-mortality associations may be influenced by other differences in behavioral factors, including degree of physical activity, smoking, alcohol consumption and diet quality, which deeply affect metabolic health independently of calorie and protein intake [10].

Here, we used data from a large Italian cohort study (EPIC-Italy, 1993-1998) of 47,749 men and women, and up to 15 years of follow-up, to prospectively examine the associations of total, animal and plant protein intake with the risk for all-cause, cardiovascular and cancer mortality. We also performed isocaloric substitution associations of different protein sources with mortality, and analyzed the joint association of protein intake and lifestyle factors (i.e., smoking status, body mass index, physical activity, alcohol consumption, and adherence to a Mediterranean dietary pattern) with disease-specific mortality. Finally, in a subset of study participants, we collected detailed data on a wide spectrum of metabolic risk factors that allowed for differential subgroup analysis of the relationship between animal and plant protein intake with key metabolic determinants of cardiovascular risk.

\section{Methods}

\subsection{Participants}

The design of the Italian section of the European Prospective Investigation into Cancer and Nutrition study [11] has been extensively described elsewhere [12]. Briefly, in EPIC-Italy a total of 47,749 healthy volunteers $(15,171$ men and 32,578 women) were recruited from 1993 to 1998 in five Italian centres (two in northern (Varese and Turin), one in central (Florence), and two in southern (Naples and Ragusa) Italy) with the aim of collecting at study entry lifestyle, medical information, and blood samples, to investigate their relationship with mortality, incident cancers, and possibly other chronic diseases. This study protocol was approved by the local Health Authority Ethics Committee in Florence, Italy. All participants provided written informed consent at enrolment and agreed to have their health status followed-up throughout life.

In the present analysis, we excluded participants with missing dietary $(n=874)$ and anthropometric $(n=361)$ data, participants lost to follow-up ( $n=$ 1052), and participants in the highest or lowest $1 \%$ of the ratio of total energy intake/basal metabolic rate $(n=449)$. After the exclusions, data from 45,009 subjects were available for the analysis.

Information on vital status was retrieved annually from municipal registries, while the cause and date of death was obtained from the official mortality indexes. Mortality data were coded according to the International Statistical Classification of Diseases and Related Health Problems, Tenth Revision (ICD-10). Deaths were grouped into common causes: cancer (ICD-10 codes: C00-D48), circulatory diseases (ICD-10 codes: I10-I25, I46-I50, I60-I69 and I70-I79). End of follow-up was December 2009 for Varese, December 2010 for Florence, Turin, Ragusa and Naples. 


\subsection{Exposure assessment}

Dietary intake was assessed only at recruitment by means of center-specific validated Food Frequency Questionnaires [FFQ) designed to better capture local eating habits, as described elsewhere [13].

Nutrient values for all food items were obtained from Italian food composition tables [14]. Total, animal and vegetable proteins were expressed as a percentage of total energy intake. Processed and unprocessed red meat, poultry, fish, egg and dairy products were the main sources of animal protein, whereas bread, cereals, pasta, nuts, and legumes were the main food contributors to vegetable protein. Animal protein by food sources was calculated as the sum of the the products between protein of each food item consumed, multiplied by the average daily amount consumed.

Adherence to a Mediterranean diet was assessed using the Italian Mediterranean Index [15], whose score is calculated from the intake of 11 items: high intake of six typical Mediterranean foods [pasta, typical Mediterranean vegetables (raw tomatoes, non-brassica leafy vegetables, courgettes, aubergines, peppers, onion and garlic), fruit, pulses, olive oil, and fish]; low intakes of four "nonMediterranean" foods (soft drinks, butter, red and processed meat, and potatoes) and moderate consumption of alcohol.

At recruitment, body weight, height (without shoes), waist and hip circumference, and blood pressure were measured according to standardized procedures [12]. Moreover, behavioural and health related variables were collected using a standardized lifestyle questionnaire, assessing reproductive history, physical activity, tobacco smoke, history of previous and/or current illnesses, medical and surgical treatment and hospitalization, education, socioeconomic status, and professional exposure. Physical activity was categorized according to the Cambridge Physical Activity Index [16].

\subsection{Blood chemistries}

Venous blood was collected after an overnight fast in a subset of 2,402 study participants randomly sampled from four Italian EPIC centers (Varese, Turin, Naples and Ragusa) [17]. Plasma glucose, lipid and lipoprotein-cholesterol concentrations were determined with automated enzymatic colorimetric commercial kits (Instrumentation Laboratory) in a previous case-cohort study [17].
High-density lipoprotein cholesterol (HDLc) was measured in plasma after precipitation of apolipoprotein B-containing lipoproteins by dextran sulfate (50000 MW) and magnesium. Low-density lipoprotein cholesterol (LDLc) was calculated using the Friedewald Equation. Non-HDL cholesterol (nonHDLc) was calculated as total cholesterol minus HDLc, while Remnant-Cholesterol was calculated as total cholesterol minus LDLc, minus HDLc. Fasting insulin was measured using ELISA kits (DRG Instruments $\mathrm{GmbH}$, Germany) and high-sensitivity C-reactive protein by a latex particle-enhanced immunoturbidimetric assay (IL Coagulation Systems on ACL9000). HOMA-IR was calculated as fasting glucose divided by fasting insulin, and $B$-cell function using HOMA- $\beta$ as fasting plasma insulin multiplied by 360 and divided by fasting plasma glucose minus 63 [18].

\subsection{Statistical analysis}

Baseline characteristics are presented by quintiles of percent of energy intake from animal and vegetable protein intake using mean and standard deviation for continuous variables and percentages for categorical variables.

We used semi-parametric Cox proportional hazards regression models with age as the time scale to estimate the hazard ratio (HR) and 95\% Confidence Interval (CI) for cause-specific and total mortality associated with total, animal and plant protein consumption.

We applied a nutrient density model with adjustment for total energy intake and the percentage of energy from fat and nonalcoholic energy. Accordingly, the coefficient for animal and plant protein reflects the substitution effect of an equal amount of energy from protein for carbohydrate (i.e., the substitution of $3 \%$ of energy from carbohydrate with an equivalent amount of energy from total, or animal or vegetable protein) in an isocaloric diet. We reported risk estimates for men and women separately. For all regression analyses sex, age, and regional center were considered as confounding factors (model 1). In the multivariable analysis, we further adjusted for several potential dietary and lifestyle confounding factors, including smoking status (current: 1-15 cig/day, 16-25 cig/day, $26+$ cig/day; former: quit $\leq 10$ years, 11-20 years, $20+$ years previously; never), physical activity (inactive, moderately inactive, moderately active, active), body mass index (BMI $\mathrm{kg} / \mathrm{m}^{2}$ ), waist 
to hip ratio, years of education $(<8$ years/ $\geq 8$ yearsmandatory school years) and alcohol, fibre, total fat [g/day) and energy (kcal/day) intake (model 2). To assess the significance of trends we employed orthogonal polynomial contrasts.

Then, we estimated the effect of substituting 3\% of energy from vegetable protein for an equivalent amount of animal protein from various sources, including processed and unprocessed red meat, poultry, fish, egg, and dairy products, by simultaneously including these protein items as continuous variables in the multivariable model. The HRs and $95 \%$ CIs for the isoprotein substitution effect in this model were derived from the difference between the regression coefficients, variance, and covariance.

Finally, to minimize the confounding effect of an overall lifestyle pattern, we further performed a stratified analysis according to body mass index $(<25$ and $\geq 25 \mathrm{~kg} / \mathrm{m}^{2}$ ), and a priori-defined healthy lifestyle pattern, constructed by assigning scores to each of the lifestyle risk factors, for which higher values indicate healthier lifestyle behaviour : low or high body mass index $\left(\geq 18.5\right.$ and $\left.<25 \mathrm{~kg} / \mathrm{m}^{2}\right)$, never smoking or ever smoking, never or moderate alcohol drinking $(<24 \mathrm{~g} / \mathrm{d})$, moderately active or active, and adherence to a Mediterranean diet defined as a Mediterranean score above the median. Tests for heterogeneity were performed adding appropriate interaction terms to the models, and testing for significance using a Wald chisquare test. Data were analyzed using Stata software (version 16.0, Stata Corp, College Station, TX, USA).

\section{Results}

After a median time of observation of 15.2 years with 644,254 person-years of follow-up, we documented 2,449 deaths, of which 1414 were due to cancer, and 441 were due to cardio- and cerebrovascular diseases. Table 1 shows the baseline characteristics of participants according to quintiles of animal and vegetable protein intake expressed as percent of energy. Mean energy from animal protein varied from 6.4 to $14 \%$ across quintiles that it is markedly lower than the amounts consumed by participants enrolled in large cohort studies of North America [Supplementary Table 1); in contrast, mean energy from vegetable protein was substantially similar ranging from $3.4 \%$ (lowest quintile) to $7.1 \%$ (highest quintile). Compared with participants in the lowest energy from animal protein quintile, those in the highest quintile consumed more fat, and less vegetable protein, starch, sugar, alcohol and energy; they had higher BMI and lower waist-to-hip ratio, and were less educated and physically active. Participants in the highest quintile of energy from animal proteins consumed more protein from processed and unprocessed red meat, poultry, egg and dairy but less pasta, bread and pizza. In contrast, those with higher plant protein intake consumed less fat, sugar and alcohol, and more starch and energy, than those in lowest quintile. Similarly to the participants in the highest quintile of energy from animal, they had higher BMI and were less educated. Participants in the highest quintile of energy from vegetable proteins were more often smokers, physically inactive, and consumed more protein from fish, and calories from pasta, bread, pizza, and legumes. Vegetable protein consumption was higher in southern Italy (Napoli and Ragusa), whereas animal protein intake was greater in northern and central Italy (Turin, Varese and Florence).

Participants with higher intake of energy from animal protein had significantly higher total, LDL and non-HDL cholesterol $(p=0.0001)$, and fasting glucose $(p=0.008)$, despite consuming significantly less calories. In contrast, those with the highest intake of energy from vegetable protein had lower total cholesterol $(p=0.001)$, LDLc $(p=0.029)$, HDLc $(p=0.001)$, fasting insulin $(p=0.01)$ but higher triglycerides $(p=0.003)$, triglyceride/HDL ratio $[p=0.001)$, remnant-cholesterol $(p=0.003)$, and C-reactive protein $(p=0.021)$ than participants in the lowest quintile (Table 1 and supplementary Table 2). Adjustment for saturated intake did not change the results for total cholesterol and LDL cholesterol (data not shown).

As shown in Table 2, no association was observed between total, animal and vegetable protein intake and all-cause, cancer or cardiovascular mortality when energy from protein was substituted with carbohydrates. These results were similar in both sexes with a $\mathrm{p}$ for interaction of 0.577 for animal protein intake and of 0.612 for vegetable protein. Table 3 shows HRs for all-cause and cause-specific mortality associated with iso-proteic replacement of 3\% of energy from animal protein and animal protein sources with plant protein. A reduction in all-cause of mortality risk was observed when substituting processed meat protein for vegetable protein in the crude (HR $0.77,95 \%$ CI $0.61-0.97$ ) but not in the fully adjusted model (HR $0.89,95 \%$ CI $0.71-1.12$ ). Similarly, a risk reduction for overall and CVD 
Table 1

Characteristics of Study Participants According to Percentage of Energy From Protein Intake ${ }^{\S}$

\begin{tabular}{|c|c|c|c|c|c|c|c|}
\hline & & \multicolumn{3}{|c|}{ Animal protein } & \multicolumn{3}{|c|}{ Vegetable protein } \\
\hline & & Quintile 1 & Quintile 3 & Quintile 5 & Quintile 1 & Quintile 3 & Quintile 5 \\
\hline \multicolumn{8}{|l|}{ Characteristics } \\
\hline Subjects & 43,213 & 8643 & 8642 & 8642 & 8643 & 8642 & 8642 \\
\hline Age & $50.7[7.9]$ & $50.0[8.14]$ & $50.7[7.92]$ & $51.6[7.66]$ & $50.7[7.90]$ & $50.6[7.94]$ & 51.0 [7.90] \\
\hline \multicolumn{8}{|l|}{ Gender } \\
\hline Male & 12.8 & 25.5 & 20.1 & 13.9 & 15.9 & 21.8 & 20.6 \\
\hline Female & 30.4 & 17.7 & 20.0 & 22.6 & 21.7 & 19.2 & 19.8 \\
\hline \multicolumn{8}{|l|}{ Center } \\
\hline Turin & 20.9 & 15.7 & 20.1 & 23.2 & 24.0 & 22.9 & 10.3 \\
\hline Varese & 26.3 & 13.9 & 21.6 & 24.7 & 33.1 & 20.8 & 5.5 \\
\hline Florence & 27.8 & 16.7 & 20.0 & 23.3 & 18.9 & 22.4 & 15.2 \\
\hline Naples & 11.4 & 18.5 & 22.9 & 13.1 & 1.7 & 11.3 & 57.5 \\
\hline Ragusa & 13.5 & 46.7 & 14.3 & 5.02 & 6.00 & 16.3 & 41.3 \\
\hline BMI $\left[\mathrm{Kg} / \mathrm{m}^{2}\right]$ & $26.0[4.1]$ & $25.6[3.96]$ & 25.9 [4.07] & $26.6[4.24]$ & $25.7[4.04]$ & $25.9[4.04]$ & $26.6[4.28]$ \\
\hline Waist to hip ratio & $0.84[0.09]$ & $0.85[0.09]$ & $0.83[0.09]$ & $0.83[0.09]$ & $0.82[0.09]$ & $0.84[0.09]$ & $0.85[0.009]$ \\
\hline Current smoker [\%] & 20.1 & 21.5 & 19.5 & 18.6 & 19.7 & 19.3 & 22.0 \\
\hline \multicolumn{8}{|l|}{ Physical activity } \\
\hline Inactive [\%] & 30.0 & 20.2 & 20.5 & 19.8 & 14.8 & 17.6 & 30.9 \\
\hline Moderately inactive [\%] & 38.2 & 18.1 & 20.3 & 22.0 & 23.0 & 21.2 & 14.7 \\
\hline Moderately active [\%] & 17.4 & 20.3 & 20.3 & 18.8 & 21.3 & 21.2 & 15.4 \\
\hline Active $[\%]$ & 14.3 & 24.3 & 19.2 & 17.0 & 21.2 & 20.4 & 16.9 \\
\hline Education [ $>$ 8years] & 22.0 & 20.4 & 20.4 & 18.7 & 19.3 & 20.6 & 18.5 \\
\hline Diastolic Pressure & $82[10]$ & $81[9.8]$ & $82[10]$ & $83[10]$ & $82[10]$ & $82[10]$ & $81.0[10]$ \\
\hline Systolic Pressure & $129[18]$ & $128[18]$ & $130[18]$ & $131[19]$ & $129[18]$ & $129[18]$ & $130[19]$ \\
\hline \multicolumn{8}{|l|}{ Dietary intake } \\
\hline $\begin{array}{l}\text { Total protein } \\
{[\% \text { energy/day }]}\end{array}$ & $16.8[2.40]$ & $14.0[1.35]$ & $16.6[0.98]$ & $20.0[1.74]$ & $17.2[2.77]$ & $16.9[2.34]$ & 16.2 [1.99] \\
\hline $\begin{array}{l}\text { Animal protein } \\
\text { [\% energy/day] }\end{array}$ & $10.1[2.84]$ & $6.41[1.16]$ & $9.97[0.40]$ & $14.3[1.81]$ & $11.7[3.09]$ & $10.4[2.47]$ & $8.12[2.23]$ \\
\hline $\begin{array}{l}\text { Vegetable protein } \\
{[\% \text { energy/day }]}\end{array}$ & $5.1[1.29]$ & $6.01[1.45]$ & $5.10[1.13]$ & $4.36[0.96]$ & $3.45[0.50]$ & $5.05[0.18]$ & $7.06[0.73]$ \\
\hline Total fat [\% energy/day] & $34.1[5.70]$ & $30.4[5.76]$ & $34.1[4.81]$ & $37.9[5.16]$ & $38.0[5.34]$ & $34.5[4.87]$ & 29.6 [4.79] \\
\hline Starch [\% energy/day] & $27.5[7.47]$ & $33.1[7.75]$ & $27.4[6.17]$ & $22.0[5.84]$ & $19.7[4.87]$ & $26.9[4.19]$ & $36.7[5.72]$ \\
\hline Sugar [\% energy/day] & $17.6[5.40]$ & $18.3[6.05]$ & $17.7[5.24]$ & $16.7[4.96]$ & $20.4[5.94]$ & $17.4[4.91]$ & $15.0[4.43]$ \\
\hline Alcohol [\% energy/day] & $3.92[4.97]$ & $4.2[5.4]$ & $4.1[5.0]$ & $3.3[4.5]$ & $4.7[6.0]$ & $4.2[4.9]$ & $2.5[3.5]$ \\
\hline Fiber $[\mathrm{g} /$ day] & $22.3[7.63]$ & $26.0[8.8]$ & $22.3[6.9]$ & $18.3[5.9]$ & $18.3[6.3]$ & $22.2[7.2]$ & $26.5[8.0]$ \\
\hline $\begin{array}{l}\text { Total energy } \\
\text { intake [kcal/day] }\end{array}$ & $2297[655]$ & $2446[694]$ & $2327[630]$ & $2049[603]$ & $2250[677]$ & $2291[665]$ & $2347[632]$ \\
\hline \multicolumn{8}{|l|}{ Protein Sources } \\
\hline Red Meat [\% energy/day] & $2.6[1.6]$ & $1.4[0.8]$ & $2.5[1.0]$ & 3.9 [1.9] & $2.9[1.8]$ & $2.7[1.5]$ & $2.0[1.8]$ \\
\hline $\begin{array}{l}\text { Processed meat } \\
{[\% \text { energy/day }]}\end{array}$ & $1.0[0.8]$ & $0.6[0.5]$ & $1.0[0.7]$ & $1.3[1.0]$ & $1.2[0.9]$ & $1.0[0.7]$ & $0.7[0.6]$ \\
\hline Poultry [\% energy/day] & $1.4[1.1]$ & $0.8[0.6]$ & $1.3[0.8]$ & $2.1[1.4]$ & $1.5[1.1]$ & $1.5[1.1]$ & $1.2[0.9]$ \\
\hline Fish [\% energy/day] & $1.2[0.9]$ & $0.7[0.6]$ & $1.1[0.7]$ & $1.6[0.9]$ & $1.1[0.9]$ & $1.2[0.9]$ & $1.1[0.9]$ \\
\hline Egg [\% energy/day] & $0.41[0.27]$ & $0.33[0.23]$ & $0.40[0.25]$ & $0.49[0.32]$ & $0.46[0.30]$ & $0.42[0.26]$ & $0.34[0.23]$ \\
\hline Dairy [\% energy/day] & $3.6[1.6]$ & $2.4[1.1]$ & $3.6[1.3]$ & $4.8[1.9]$ & $4.4[1.9]$ & $3.6[1.5]$ & $2.7[1.2]$ \\
\hline Tubers [\% energy/day] & $0.10[0.08]$ & $0.10[0.09]$ & $0.10[0.08]$ & $0.10[0.08]$ & $0.10[0.08]$ & $0.11[0.08]$ & $0.11[0.08]$ \\
\hline Vegetables [\% energy/day] & $0.63[0.34]$ & $0.59[0.35]$ & $0.63[0.33]$ & $0.68[0.34]$ & $0.57[0.29]$ & $0.64[0.33]$ & $0.68[0.39]$ \\
\hline Legumes [\% energy/day] & $0.18[0.33]$ & $0.17[0.32]$ & $0.20[0.35]$ & $0.15[0.40]$ & $0.08[0.09]$ & $0.13[0.20]$ & $0.39[0.55]$ \\
\hline Fruits [\% energy/day] & $0.40[0.23]$ & $0.44[0.28]$ & $0.39[0.21]$ & $0.37[0.20]$ & $0.37[0.21]$ & $0.40[0.23]$ & $0.42[0.24]$ \\
\hline Pasta [\% energy/day] & $0.83[0.58]$ & $0.95[0.66]$ & $0.86[0.56]$ & $0.66[0.50]$ & $0.54[0.40]$ & $0.87[0.55]$ & $1.05[0.66]$ \\
\hline Rice [\% energy/day] & $0.14[0.16]$ & $0.13[0.18]$ & $0.15[0.16]$ & $0.14[0.16]$ & $0.13[0.14]$ & $0.15[0.17]$ & $0.12[0.16]$ \\
\hline Bread [\% energy/day] & $2.22[1.23]$ & $3.02[1.49]$ & $2.14[1.06]$ & $1.58[0.90]$ & $1.07[0.61]$ & $2.09[0.73]$ & $3.69[1.26]$ \\
\hline Pizza [\% energy/day] & $0.18[0.15]$ & $0.20[0.18]$ & $0.18[0.15]$ & $0.16[0.14]$ & $0.16[0.13]$ & $0.18[0.15]$ & $0.21[0.18]$ \\
\hline
\end{tabular}


Table 1

(Continued)

\begin{tabular}{|c|c|c|c|c|c|c|c|}
\hline & & \multicolumn{3}{|c|}{ Animal protein } & \multicolumn{3}{|c|}{ Vegetable protein } \\
\hline & & Quintile 1 & Quintile 3 & Quintile 5 & Quintile 1 & Quintile 3 & Quintile 5 \\
\hline \multicolumn{8}{|l|}{ PLASMA BIOMARKERS* } \\
\hline Insulin [mU/L] & $9.87[8.04]$ & $9.77[6.02]$ & $9.88[7.95]$ & $9.58[6.09]$ & $10.1[6.7]$ & $10.2[8.3]$ & $8.9[0.6]$ \\
\hline Glycemia [mg/dl] & $100.4[30.7]$ & $98.6[29.0]$ & $100[31.5]$ & $103.7[35.3]$ & $100.3[27.4]$ & $102.6[34.2]$ & 99.3 [32.7] \\
\hline HOMA-IR & $2.2[2.61]$ & $2.08[1.59]$ & $2.22[2.75]$ & $2.18[1.96]$ & $2.17[1.65]$ & $2.29[2.42]$ & $1.92[1.63]$ \\
\hline$\beta$-cell function & 207 [509] & $231[594]$ & $162[605]$ & $183[361]$ & $227[501]$ & 178 [493] & $169[632]$ \\
\hline $\mathrm{CRP}[\mathrm{mg} / \mathrm{ml}]$ & $1.99[2.63]$ & $1.93[2.30]$ & $1.94[2.53]$ & $2.06[2.43]$ & $1.80[2.23]$ & $2.17[3.05]$ & $2.20[2.62]$ \\
\hline Cholesterol $[\mathrm{mg} / \mathrm{dl}]$ & $235.3[48.0]$ & $228.4[46.8]$ & $235.9[49.4]$ & $241.6[47.5]$ & $241.0[45.7]$ & $232.6[47.3]$ & $230.3[50.4]$ \\
\hline Triglycerides $[\mathrm{mg} / \mathrm{dl}]$ & $143.7[88.2]$ & $145.4[78.0]$ & $140.2[82.1]$ & $139.3[85.9]$ & $133.1[77.3]$ & $140.1[74.8]$ & $148.0[80.1]$ \\
\hline $\mathrm{HDL}[\mathrm{mg} / \mathrm{l}]$ & $59.4[15.4]$ & $58.0[15.3]$ & $60.1[15.4]$ & $61.0[15.2]$ & $63.2[15.3]$ & $59.1[15.7]$ & $55.4[13.9]$ \\
\hline Triglyceride/HDL ratio & $2.77[2.44]$ & $2.82[1.98]$ & $2.65[2.16]$ & $2.61[2.37]$ & $2.40[2.15]$ & $2.72[2.02]$ & $2.95[2.05]$ \\
\hline Remnant-Cholesterol [mg/dl] & $28.7[17.7]$ & $29.1[15.6]$ & $28.0[16.4]$ & $27.9[17.2]$ & $26.6[15.5]$ & $28.0[15.0]$ & $29.6[16.0]$ \\
\hline Non HDLc [mg/dl] & $175.9[46.2]$ & $170.3[45.5]$ & $175.7[47.3]$ & $180.7[45.5]$ & $177.7[44.8]$ & $173.4[45.5]$ & $174.9[48.3]$ \\
\hline LDLc [mg/dl] & $147.1[41.2]$ & $141.3[39.2]$ & $147.7[42.4]$ & $152.8[41.9]$ & $151.1[40.3]$ & $145.4[40.6]$ & $145.3[42.8]$ \\
\hline
\end{tabular}

$\S$ Table entries are means and standard deviation except where indicated *Plasma metabolic and cardiovascular biomarkers were measured after an overnight fast in a subset of 2,402 study participants randomly sampled from four Italian EPIC centers [Varese, Turin, Naples and Ragusa].

Table 2

Risk for All-Cause and Cause-Specific Mortality Associated with Replacement of $3 \%$ of Energy from Protein with Carbohydrate

\begin{tabular}{|c|c|c|c|}
\hline & All cause of mortality & Cancer mortality & $\begin{array}{c}\text { Cardiovascular and } \\
\text { Cerebrovascular } \\
\text { disease mortality }\end{array}$ \\
\hline All subjects & $n$. deaths $=2,449$ & $n$. deaths $=1,414$ & $n$. deaths $=441$ \\
\hline \multicolumn{4}{|l|}{ Total protein } \\
\hline $\mathrm{HR}^{1}[95 \% \mathrm{CI}]$ & 1.03 [0.98-1.09] & 1.01 [0.94-1.09] & $1.13[0.99-1.28]$ \\
\hline $\mathrm{HR}^{3}[95 \% \mathrm{CI}]$ & $0.98[0.93-1.04]$ & $0.98[0.90-1.05]$ & $1.04[0.91-1.20]$ \\
\hline \multicolumn{4}{|l|}{ Animal protein } \\
\hline $\mathrm{HR}^{1}[95 \% \mathrm{CI}]$ & 1.01 [0.95-1.07] & 1.01 [0.93-1.09] & $1.09[0.95-1.25]$ \\
\hline $\mathrm{HR}^{3}[95 \% \mathrm{CI}]$ & $0.96[0.90-1.02]$ & $0.97[0.90-1.05]$ & $1.01[0.87-1.17]$ \\
\hline \multicolumn{4}{|l|}{ Vegetable protein } \\
\hline $\mathrm{HR}^{1}[95 \% \mathrm{CI}]$ & $0.99[0.83-1.18]$ & $0.96[0.76-1.22]$ & $0.98[0.95-1.25]$ \\
\hline $\mathrm{HR}^{3}[95 \% \mathrm{CI}]$ & $0.94[0.79-1.12]$ & $0.95[0.75-1.21]$ & $0.86[0.57-1.31]$ \\
\hline Men & $n$. deaths $=995$ & $n$. deaths $=538$ & $n$. deaths $=204$ \\
\hline \multicolumn{4}{|l|}{ Total protein } \\
\hline $\mathrm{HR}^{2}[95 \% \mathrm{CI}]$ & $1.05[0.96-1.16]$ & $1.09[0.96-1.23]$ & 1.17 [0.95-1.44] \\
\hline $\mathrm{HR}^{3}[95 \% \mathrm{CI}]$ & $1.01[0.91-1.12]$ & $1.05[0.91-1.20]$ & $1.07[0.86-1.34]$ \\
\hline \multicolumn{4}{|l|}{ Animal protein } \\
\hline $\mathrm{HR}^{2}[95 \% \mathrm{CI}]$ & $1.00[0.91-1.10]$ & $1.05[0.92-1.20]$ & $1.10[0.89-1.36]$ \\
\hline $\mathrm{HR}^{3}[95 \% \mathrm{CI}]$ & $0.97[0.88-1.08]$ & $1.04[0.90-1.20]$ & $1.00[0.79-1.26]$ \\
\hline \multicolumn{4}{|l|}{ Vegetable protein } \\
\hline $\mathrm{HR}^{2}[95 \% \mathrm{CI}]$ & $0.84[0.63-1.12]$ & $1.00[0.68-1.48]$ & $0.71[0.89-1.36]$ \\
\hline $\mathrm{HR}^{3}[95 \% \mathrm{CI}]$ & $0.78[0.59-1.05]$ & $0.96[0.65-1.43]$ & 0.57 [0.30-1.09] \\
\hline Women & $n$. deaths $=1454$ & $n$. deaths $=876$ & n. deaths $=237$ \\
\hline \multicolumn{4}{|l|}{ Total protein } \\
\hline $\mathrm{HR}^{2}[95 \% \mathrm{CI}]$ & $1.02[0.96-1.10]$ & 0.98 [0.90-1.07] & $1.12[0.94-1.33]$ \\
\hline $\mathrm{HR}^{3}[95 \% \mathrm{CI}]$ & $0.98[0.91-1.05]$ & $0.94[0.86-1.04]$ & $1.04[0.87-1.24]$ \\
\hline \multicolumn{4}{|l|}{ Animal protein } \\
\hline $\mathrm{HR}^{2}[95 \% \mathrm{CI}]$ & 1.01 [0.94-1.09] & $0.98[0.90-1.08]$ & $1.12[0.93-1.35]$ \\
\hline $\mathrm{HR}^{3}[95 \% \mathrm{CI}]$ & $0.96[0.89-1.04]$ & $0.94[0.85-1.04]$ & $1.05[0.87-1.28]$ \\
\hline \multicolumn{4}{|c|}{ Vegetable protein } \\
\hline $\mathrm{HR}^{2}[95 \% \mathrm{CI}]$ & $1.08[0.86-1.36]$ & $0.93[0.70-1.26]$ & $1.35[0.77-2.36]$ \\
\hline $\mathrm{HR}^{3}[95 \% \mathrm{CI}]$ & $1.08[0.86-1.36]$ & $0.97[0.72-1.30]$ & $1.27[0.73-2.23]$ \\
\hline
\end{tabular}

${ }^{1}$ stratified by center, age, sex; ${ }^{2}$ stratified by center, age; ${ }^{3}$ adjusted also for energy, bmi, whr, smoking, education, physical activity, fiber, alcohol, total fat intake. 
Table 3

Risk for All-Cause and Cause-Specific Mortality Associated with Replacement of 3\% of Energy From Animal Protein and Animal protein sources with Plant Protein

\begin{tabular}{|c|c|c|c|}
\hline & All cause of mortality & Cancer mortality & $\begin{array}{c}\text { Cardiovascular and } \\
\text { Cerebrovascular } \\
\text { disease mortality }\end{array}$ \\
\hline All subjects & $n$. deaths $=2,449$ & $n \cdot$ deaths $=1,414$ & $n$. deaths $=441$ \\
\hline \multicolumn{4}{|l|}{ Animal protein } \\
\hline $\mathrm{HR}^{1}[95 \% \mathrm{CI}]$ & $0.98[0.84-1.15]$ & $0.96[0.77-1.18]$ & $0.90[0.62-1.32]$ \\
\hline $\mathrm{HR}^{2}[95 \% \mathrm{CI}]$ & $0.98[0.83-1.15]$ & $0.98[0.79-1.22]$ & $0.85[0.58-1.25]$ \\
\hline \multicolumn{4}{|l|}{ Animal sources } \\
\hline \multicolumn{4}{|l|}{ Processed meat } \\
\hline $\mathrm{HR}^{1}[95 \% \mathrm{CI}]$ & $0.77[0.61-0.97]$ & $0.80[0.59-1.07]$ & 0.64 [0.38-1.09] \\
\hline $\mathrm{HR}^{2}[95 \% \mathrm{CI}]$ & $0.89[0.71-1.12]$ & $0.92[0.68-1.25]$ & $0.76[0.44-1.30]$ \\
\hline \multicolumn{4}{|l|}{ Red meat } \\
\hline $\mathrm{HR}^{1}[95 \% \mathrm{CI}]$ & $0.98[0.82-1.18]$ & $0.99[0.78-1.26]$ & $0.73[0.48-1.12]$ \\
\hline $\mathrm{HR}^{2}[95 \% \mathrm{CI}]$ & $1.00[0.83-1.21]$ & $1.04[0.81-1.33]$ & $0.72[0.47-1.10]$ \\
\hline \multicolumn{4}{|l|}{ Poultry } \\
\hline $\mathrm{HR}^{1}[95 \% \mathrm{CI}]$ & $1.05[0.85-1.28]$ & $1.06[0.81-1.39]$ & $0.82[0.52-1.31]$ \\
\hline $\mathrm{HR}^{2}[95 \% \mathrm{CI}]$ & $0.98[0.80-1.21]$ & $1.00[0.77-1.31]$ & $0.76[0.48-1.22]$ \\
\hline \multicolumn{4}{|l|}{ Fish } \\
\hline $\mathrm{HR}^{1}[95 \% \mathrm{CI}]$ & $0.88[0.71-1.10]$ & $0.87[0.65-1.15]$ & $0.74[0.45-1.22]$ \\
\hline $\mathrm{HR}^{2}[95 \% \mathrm{CI}]$ & $0.88[0.71-1.09]$ & $0.87[0.66-1.16]$ & $0.70[0.42-1.15]$ \\
\hline \multicolumn{4}{|l|}{ Egg } \\
\hline $\mathrm{HR}^{1}[95 \% \mathrm{CI}]$ & $0.91[0.56-1.47]$ & $1.31[0.69-2.50]$ & $0.42[0.14-1.28]$ \\
\hline $\mathrm{HR}^{2}[95 \% \mathrm{CI}]$ & $0.98[0.60-1.60]$ & $1.46[0.76-2.80]$ & $0.43[0.14-1.34]$ \\
\hline \multicolumn{4}{|l|}{ Dairy } \\
\hline $\mathrm{HR}^{1}[95 \% \mathrm{CI}]$ & 1.01 [0.86-1.19] & $0.96[0.77-1.19]$ & $1.05[0.71-1.54]$ \\
\hline $\mathrm{HR}^{2}[95 \% \mathrm{CI}]$ & $1.00[0.84-1.18]$ & $0.98[0.79-1.23]$ & $0.97[0.65-1.45]$ \\
\hline Men & $n \cdot$ deaths $=995$ & n. deaths $=538$ & $n \cdot$ deaths $=204$ \\
\hline \multicolumn{4}{|l|}{ Animal protein } \\
\hline $\mathrm{HR}^{3}[95 \% \mathrm{CI}]$ & $0.84[0.65-1.09]$ & $0.95[0.66-1.36]$ & $0.65[0.36-1.16]$ \\
\hline $\mathrm{HR}^{2,3}[95 \% \mathrm{CI}]$ & $0.80[0.61-1.05]$ & $0.93[0.64-1.34]$ & $0.58[0.32-1.04]$ \\
\hline \multicolumn{4}{|l|}{ Animal sources } \\
\hline \multicolumn{4}{|l|}{ Processed meat } \\
\hline $\mathrm{HR}^{3}[95 \% \mathrm{CI}]$ & $0.65[0.45-0.93]$ & $0.71[0.44-1.16]$ & $0.42[0.19-0.91]$ \\
\hline $\mathrm{HR}^{2,3}[95 \% \mathrm{CI}]$ & $0.77[0.53-1.11]$ & $0.85[0.51-1.39]$ & $0.48[0.22-1.07]$ \\
\hline \multicolumn{4}{|l|}{ Red meat } \\
\hline $\mathrm{HR}^{3}[95 \% \mathrm{CI}]$ & 0.81 [0.61-1.09] & $0.92[0.62-1.38]$ & $0.56[0.30-1.05]$ \\
\hline $\mathrm{HR}^{2,3}[95 \% \mathrm{CI}]$ & $0.80[0.59-1.08]$ & $0.91[0.60-1.36]$ & $0.53[0.28-1.03]$ \\
\hline \multicolumn{4}{|l|}{ Poultry } \\
\hline $\mathrm{HR}^{3}[95 \% \mathrm{CI}]$ & $0.88[0.63-1.22]$ & $1.04[0.66-1.62]$ & 0.55 [0.27-1.09] \\
\hline $\mathrm{HR}^{2,3}[95 \% \mathrm{CI}]$ & $0.81[0.58-1.12]$ & $0.93[0.60-1.46]$ & $0.52[0.26-1.04]$ \\
\hline \multicolumn{4}{|l|}{ Fish } \\
\hline $\mathrm{HR}^{3}[95 \% \mathrm{CI}]$ & $0.74[0.52-1.06]$ & $1.00[0.62-1.63]$ & $0.50[0.24-1.06]$ \\
\hline $\mathrm{HR}^{2,3}[95 \% \mathrm{CI}]$ & $0.74[0.52-1.05]$ & $1.01[0.62-1.65]$ & $0.43[0.20-0.91]$ \\
\hline \multicolumn{4}{|l|}{ Egg } \\
\hline $\mathrm{HR}^{3}[95 \% \mathrm{CI}]$ & $1.27[0.54-2.99]$ & $1.96[0.61-6.30]$ & $1.00[0.16-6.42]$ \\
\hline $\mathrm{HR}^{2,3}[95 \% \mathrm{CI}]$ & $1.32[0.55-3.16]$ & $1.92[0.58-6.32]$ & $1.12[0.16-7.76]$ \\
\hline \multicolumn{4}{|l|}{ Dairy } \\
\hline $\mathrm{HR}^{3}[95 \% \mathrm{CI}]$ & $0.89[0.67-1.16]$ & $0.98[0.67-1.42]$ & 0.78 [0.43-1.42] \\
\hline $\mathrm{HR}^{2,3}[95 \% \mathrm{CI}]$ & 0.82 [0.62-1.09] & $0.94[0.64-1.38]$ & 0.68 [0.36-1.26] \\
\hline Women & $n$. deaths $=1454$ & $n$. deaths $=876$ & $n$. deaths $=237$ \\
\hline \multicolumn{4}{|l|}{ Animal protein } \\
\hline $\mathrm{HR}^{3}[95 \% \mathrm{CI}]$ & $1.07[0.87-1.31]$ & $0.95[0.73-1.24]$ & $1.20[0.73-1.98]$ \\
\hline $\mathrm{HR}^{2,3}[95 \% \mathrm{CI}]$ & $1.12[0.91-1.39]$ & $1.02[0.78-1.35]$ & $1.21[0.72-2.02]$ \\
\hline
\end{tabular}


(Continued)

\begin{tabular}{|c|c|c|c|}
\hline & All cause of mortality & Cancer mortality & $\begin{array}{c}\text { Cardiovascular and } \\
\text { Cerebrovascular } \\
\text { disease mortality }\end{array}$ \\
\hline \multicolumn{4}{|l|}{ Animal sources } \\
\hline \multicolumn{4}{|l|}{ Processed meat } \\
\hline $\mathrm{HR}^{3}[95 \% \mathrm{CI}]$ & $0.85[0.64-1.14]$ & $0.84[0.58-1.23]$ & $0.93[0.45-1.92]$ \\
\hline $\mathrm{HR}^{2,3}[95 \% \mathrm{CI}]$ & $0.99[0.74-1.34]$ & $0.97[0.66-1.43]$ & $1.19[0.57-2.51]$ \\
\hline \multicolumn{4}{|l|}{ Red meat } \\
\hline $\mathrm{HR}^{3}[95 \% \mathrm{CI}]$ & $1.10[0.87-1.40]$ & $1.03[0.76-1.41]$ & $0.94[0.53-1.68]$ \\
\hline $\mathrm{HR}^{2,3}[95 \% \mathrm{CI}]$ & $1.19[0.93-1.51]$ & $1.15[0.84-1.57]$ & $0.99[0.56-1.77]$ \\
\hline \multicolumn{4}{|l|}{ Poultry } \\
\hline $\mathrm{HR}^{3}[95 \% \mathrm{CI}]$ & $1.16[0.89-1.50]$ & $1.05[0.75-1.47]$ & $1.20[0.63-2.25]$ \\
\hline $\mathrm{HR}^{2,3}[95 \% \mathrm{CI}]$ & $1.14[0.88-1.47]$ & $1.05[0.75-1.47]$ & $1.14[0.60-2.12]$ \\
\hline \multicolumn{4}{|l|}{ Fish } \\
\hline $\mathrm{HR}^{3}[95 \% \mathrm{CI}]$ & $0.98[0.75-1.29]$ & $0.81[0.57-1.14]$ & $1.03[0.52-2.02]$ \\
\hline $\mathrm{HR}^{2,3}[95 \% \mathrm{CI}]$ & $1.00[0.76-1.31]$ & $0.83[0.59-1.18]$ & $1.12[0.57-2.21]$ \\
\hline \multicolumn{4}{|l|}{ Egg } \\
\hline $\mathrm{HR}^{3}[95 \% \mathrm{CI}]$ & $0.79[0.44-1.41]$ & $1.08[0.50-2.34]$ & $0.26[0.07-1.03]$ \\
\hline $\mathrm{HR}^{2,3}[95 \% \mathrm{CI}]$ & $0.87[0.48-1.58]$ & $1.33[0.61-2.92]$ & $0.25[0.06-0.99]$ \\
\hline \multicolumn{4}{|l|}{ Dairy } \\
\hline $\mathrm{HR}^{3}[95 \% \mathrm{CI}]$ & $1.09[0.88-1.34]$ & $0.94[0.72-1.23]$ & $1.35[0.81-2.26]$ \\
\hline $\mathrm{HR}^{2,3}[95 \% \mathrm{CI}]$ & $1.14[0.92-1.42]$ & $1.02[0.77-1.35]$ & $1.36[0.80-2.31]$ \\
\hline
\end{tabular}

${ }^{1}$ stratified by center, age, sex. ${ }^{2}$ adjusted also for energy, bmi, whr, smoking, education, physical activity, fiber, alcohol, total fat intake.

${ }^{3}$ stratified by center, age.

mortality was observed in men in the crude model when substituting processed meat protein for vegetable protein (HR 0.65, 95\% CI 0.45-0.93; HR 0.42, 95\% CI 0.19-0.91, respectively), but not in the fully adjusted model. A significant reduction in CVD mortality was observed in men only, even in the fully adjusted model, when fish protein was substituted for vegetable protein (HR $0.43,95 \%$ CI $0.20-0.91$ ). In women, CVD mortality was significantly reduced by substituting egg protein for vegetable protein (HR $0.25,95 \%$ CI $0.06-0.99$ in the fully adjusted model).

When we analyzed associations between animal and vegetable protein and mortality risk by subgroups of BMI category ( $<25$ and $>25 \mathrm{~kg} / \mathrm{m}^{2}$ ), waist-to-hip ratio, or age, no significant interaction was found (data not shown). However, when we categorized participants into healthy- and unhealthy-lifestyle groups according to a priori-defined criteria (Table 4), we found a protective association with CVD mortality of substituting vegetable protein for carbohydrate that was restricted only to participants with at least 1 unhealthy lifestyle risk factor and poor adherence to the Mediterranean diet (HR 0.45, 95\% CI 0.22-0.94). Similarly, in the unhealthy-lifestyle group but not in the healthy lifestyle group, the multivariable HR per $3 \%$ replacement of animal with vegetable protein was 0.47 (95\% CI, 0.24-0.92).

\section{Discussion}

In this large cohort study of 45,009 Italian men and women who were followed for a mean of 15.2 years, we observed no clear significant association between total or animal protein intake and total or causespecific mortality. However, a higher plant protein consumption was associated with a $\sim 50 \%$ lower risk of cardiovascular disease mortality but only in participants with at least 1 unhealthy lifestyle risk factor and poor adherence to the Mediterranean diet. High vegetable protein intake was associated with significantly lower plasma cholesterol and insulin levels, while high animal protein was associated with elevated plasma cholesterol and fasting glucose, despite consuming significantly less calories and having similar BMIs, suggesting that protein source plays crucial roles in modulating cardiometabolic health independently of body weight.

Adherence to high protein diets, especially animal protein rich in branched-chain amino acids and sulfur amino acids, has become very popular because of its hypothetical role in preserving muscle mass, and in promoting fat loss and associated improvements in cardiometabolic health [19-22]. However, conclusive evidence that consuming high protein diets has meaningful effects on body composition or metabolic 
Table 4

Risk for Total, Cancer and CVD Mortality Associated with Replacement of $3 \%$ of Energy From Protein with Carbohydrate and Animal Protein with Vegetable Protein Among Participants with Healthy and Unhealthy Lifestyles

\begin{tabular}{|c|c|c|c|}
\hline & All cause of mortality & Cancer mortality & $\begin{array}{c}\text { Cardiovascular and } \\
\text { Cerebrovascular } \\
\text { disease mortality }\end{array}$ \\
\hline \multicolumn{4}{|l|}{ Total protein } \\
\hline \multicolumn{4}{|l|}{ Healthy $^{\mathrm{a}}$} \\
\hline $\mathrm{HR}^{1}[95 \% \mathrm{CI}]$ & $1.04[0.97-1.11]$ & $1.01[0.92-1.10]$ & $1.12[0.95-1.33]$ \\
\hline $\mathrm{HR}^{2}[95 \% \mathrm{CI}]$ & $0.98[0.91-1.06]$ & $0.95[0.87-1.05]$ & $1.03[0.86-1.22]$ \\
\hline \multicolumn{4}{|l|}{ Unhealthy $^{\mathrm{b}}$} \\
\hline $\mathrm{HR}^{1}[95 \% \mathrm{CI}]$ & $1.02[0.93-1.12]$ & $1.02[0.91-1.15]$ & $1.12[0.90-1.40]$ \\
\hline $\mathrm{HR}^{2}[95 \% \mathrm{CI}]$ & $0.97[0.88-1.07]$ & $0.99[0.87-1.12]$ & $1.02[0.80-1.29]$ \\
\hline \multicolumn{4}{|l|}{ Animal protein } \\
\hline \multicolumn{4}{|l|}{ Healthy $y^{\mathrm{a}}$} \\
\hline $\mathrm{HR}^{1}[95 \% \mathrm{CI}]$ & 1.01 [0.94-1.09] & $0.99[0.90-1.09]$ & $1.11[0.93-1.32]$ \\
\hline $\mathrm{HR}^{2}[95 \% \mathrm{CI}]$ & $0.96[0.88-1.03]$ & $0.94[0.85-1.04]$ & $1.01[0.84-1.21]$ \\
\hline \multicolumn{4}{|l|}{ Unhealthy $^{\mathrm{b}}$} \\
\hline $\mathrm{HR}^{1}[95 \% \mathrm{CI}]$ & $0.99[0.90-1.10]$ & $1.03[0.91-1.17]$ & $1.03[0.82-1.31]$ \\
\hline $\mathrm{HR}^{2}[95 \% \mathrm{CI}]$ & $0.93[0.84-1.04]$ & $0.99[0.86-1.13]$ & $0.95[0.74-1.23]$ \\
\hline \multicolumn{4}{|c|}{ Vegetable protein } \\
\hline \multicolumn{4}{|c|}{ Healthy $y^{\mathrm{a}}$} \\
\hline $\mathrm{HR}^{1}[95 \% \mathrm{CI}]$ & $0.99[0.80-1.24]$ & $0.87[0.65-1.17]$ & $1.31[0.77-2.21]$ \\
\hline $\mathrm{HR}^{2}[95 \% \mathrm{CI}]$ & $0.98[0.79-1.23]$ & $0.89[0.67-1.20]$ & $1.23[0.73-2.07]$ \\
\hline \multicolumn{4}{|l|}{ Unhealthy $^{\mathrm{b}}$} \\
\hline $\mathrm{HR}^{1}[95 \% \mathrm{CI}]$ & $0.86[0.63-1.18]$ & $0.98[0.65-1.48]$ & $0.57[0.28-1.16]$ \\
\hline $\mathrm{HR}^{2}[95 \% \mathrm{CI}]$ & $0.77[0.57-1.08]$ & $0.92[0.61-1.40]$ & $0.45[0.22-0.94]$ \\
\hline \multicolumn{4}{|c|}{$\begin{array}{l}\text { Animal protein for } \\
\text { vegetable protein }\end{array}$} \\
\hline \multicolumn{4}{|l|}{ Healthy ${ }^{\mathrm{a}}$} \\
\hline $\mathrm{HR}^{1}[95 \% \mathrm{CI}]$ & 0.99 [0.80-1.21] & $0.88[0.67-1.15]$ & $1.18[0.73-1.91]$ \\
\hline $\mathrm{HR}^{2}[95 \% \mathrm{CI}]$ & $1.03[0.83-1.26]$ & $0.95[0.72-1.25]$ & $1.22[0.75-1.99]$ \\
\hline \multicolumn{4}{|l|}{ Unhealthy $^{\mathrm{b}}$} \\
\hline $\mathrm{HR}^{1}[95 \% \mathrm{CI}]$ & $0.87[0.65-1.15]$ & $0.95[0.66-1.38]$ & $0.55[0.29-1.04]$ \\
\hline $\mathrm{HR}^{2}[95 \% \mathrm{CI}]$ & $0.84[0.63-1.12]$ & $0.93[0.64-1.36]$ & $0.47[0.24-0.92]$ \\
\hline
\end{tabular}

${ }^{1}$ stratified by center, age, sex; ${ }^{2}$ adjusted also for energy, bmi, whr, smoking, education, physical activity, fiber, alcohol, total fat intake. ${ }^{a}$ Healthy lifestyle was defined as never smoking, never or moderate alcohol intake $[<24 \mathrm{~g} /$ day $]$, physically active, BMI between 18.5 and $25 \mathrm{~kg} / \mathrm{m}^{2}$ and adherence to Mediterranean diet. ${ }^{\mathrm{b}}$ Unhealthy lifestyle was defined as having at least 1 of these unhealthy lifestyle risk factors [current smoker, alcohol intake in excess of $24 \mathrm{~g} /$ day, BMI $\geq 25 \mathrm{~kg} / \mathrm{m}^{2}$, sedentary lifestyle] and poor adherence to the Mediterranean diet.

health is controversial, and accumulating evidence suggest it might have adverse effects on insulin sensitivity, and it may increase the risk of developing obesity, type 2 diabetes, and therefore cardiovascular disease [23].

Branched-chain amino acids are known activators of the mammalian target of rapamycin complex 1 [mTORC1) pro-aging signaling pathway that is deeply implicated in the regulation of lipid/glucose metabolism, protein turnover, autophagy, and nucleotide and ribosomal biogenesis [24,25]. On the other end, non-essential amino acids, which predominate in plant proteins, stimulate glucagon secretion [26]. Glucagon can promote a cAMP-dependent downregulation of lipogenic enzymes and cholesterol synthesis, while up-regulating hepatic LDL receptors and IGFBP-1 production in the liver [27]. A lower sulfur amino acid intake, especially of methionine, increases longevity in rodents, and has been associated with reduced risk for cardiometabolic diseases in a recent analysis from the NHANES III Study [28]. As methionine is specified by the AUG start codon, it is essential for translation initiation of most proteins. Methionine restriction thus has a dramatic effect in downregulating protein synthesis and activating FGF21, a key regulator of insulin sensitivity and energy expenditure [25, 29].

Our current findings together with the results of other epidemiological studies suggest that replacement of foods high in animal protein with plant protein sources, but not total protein intake per se, is associated with lower cardiovascular mortality [5, 7, 30-32]. However, unlike in other North American studies of people with substantially higher intake of 
animal food, we did not find an association between animal protein and cardiovascular mortality. Moreover, our data confirm that even in this cohort of Italian consuming less animal protein, the protective effect of vegetable protein is confined only to participants with unhealthy diets, and one or more negative lifestyle risk factors.

There are multiple explanations for these findings. Indeed, residual confounding from metabolic factors may contribute to the observed protein-mortality associations even after adjustment for age, sex, BMI, energy intake, smoking, physical activity, fiber, alcohol, and total fat intake. At least eighty per cent of cardiovascular disease risk and mortality can be explained by the additive effects of classical cardiovascular risk factors [33-35]. Therefore, when evaluating the relation between protein intake and mortality, it might be important to consider their interactive and potentially counteractive actions. Interestingly, in our study both higher animal and plant protein intakes were associated with similar increase in BMI. However, only animal protein consumption was strongly associated with increased fasting glucose and LDLc, whereas high vegetable protein was associated with significantly lower LDLc and fasting insulin, and a trend for lower fasting glucose as well. This is in agreement with recent data from a randomized clinical trial demonstrating that increasing protein intake from 0.8 to $1.2 \mathrm{~g} / \mathrm{kg}$ per day by adding whey protein rich in branched-chain amino acids completely prevented the beneficial effect of visceral and liver fat loss induced by calorie restriction on insulin-mediated glucose disposal [36].

Our findings suggest that other components in vegetable protein-rich foods [e.g., fiber, starch), in addition to protein per se, may have a critical cardiometabolic modulatory effects. The elevated fiber content of foods rich in plant protein might explain their LDLc lowering effect because this association persisted even after adjustment for saturated fatty acid content $[37,38]$. In contrast, the high content of starch from white bread, pizza, and pasta might explain the detrimental effects of vegetable protein-rich foods on circulating remnant-cholesterol, cholesterol:HDL ratio, triglycerides and C-reactive protein levels, which may mitigate the protective effects of lower LDLc and higher insulin sensitivity [39-41]. It is possible that other phytochemicals that predominate in vegetable protein-rich foods, such as sterols, phenolics, flavonoids, saponins, sulfides and organosulfur compounds, could also affect lipid and cholesterol metabolism through multiple mechanisms, including inhibition of cholesterol biosynthesis and LDL oxidation, and reduction of cholesterol absorption by disrupting the solubility of micelles [42].

This study has strengths and weaknesses. Our analysis was performed on a large sample size of Italian men and women who were followed for 15.2 years and consumed substantially less animal protein than other cohorts of North America. This is important because our findings can be more easily translated to populations living in developing countries consuming carbohydrate-rich diets low in animal food products. The availability of a wide range of lifestyle factors allowed for rigorous confounding adjustment and subgroup analysis, whereas the cardiometabolic biomarker phenotyping provided a unique mechanistic insight into these associations. Calculation of protein intake according to food sources, and the substitution effect for protein of various origins is another strength of our study. One limitation is that dietary exposure was assessed only once at baseline. Some participants may have changed their diet during the follow-up, giving rise to potential misclassification of exposure, which could have weakened diet-disease associations. Moreover, although we have adjusted our results for several dietary and lifestyle factors, residual confounding remains a possibility because of unmeasured or unknown factors.

\section{Conclusions}

In conclusion, we found that substitution of plant protein for animal protein is associated with lower cardiovascular mortality, which is consistent with its beneficial effects on plasma cholesterol levels and glucose metabolism, especially in people with unhealthy lifestyle factors. These results have important public health implications especially when the cardiometabolic detrimental effects of consuming starch-rich plant foods can be mitigated by regular exercise training and higher intake of plant foods rich in protein and low in refined carbohydrates such as legumes.

\section{Acknowledgments and Funding}

The authors have no financial conflicts of interest to disclose. EPIC-Italy is funded by the Italian Association for Research on Cancer. L.F. is supported by grants from the Australian NHMRC Investigator 
Grant [APP1177797), Australian Youth and Health Foundation and Philip Bushell Foundation.

\section{Conflict of interest}

Luigi Fontana is an Editorial Board Member of this journal, but was not involved in the peer-review process nor had access to any information regarding its peer-review.

\section{Supplementary material}

The supplementary material is available in the electronic version of this article: https://dx.doi.org/ 10.3233/NHA-210145.

\section{References}

[1] Fontana L, Partridge L. Promoting health and longevity through diet: from model organisms to humans. Cell. 2015;161(1):106-18.

[2] Solon-Biet SM, McMahon AC, Ballard JW, Ruohonen K, Wu LE, Cogger VC, et al. The ratio of macronutrients, not caloric intake, dictates cardiometabolic health, aging, and longevity in ad libitum-fed mice. Cell Metab. 2014;19(3): 418-30.

[3] Ables GP, Brown-Borg HM, Buffenstein R, Church CD, Elshorbagy AK, Gladyshev VN, et al. The first international mini-symposium on methionine restriction and lifespan. Front Genet. 2014;5:122.

[4] Richardson NE, Konon EN, Schuster HS, Mitchell AT, Boyle $\mathrm{C}$, Rodgers $\mathrm{AC}$, et al. Lifelong restriction of dietary branchedchain amino acids has sex-specific benefits for frailty and lifespan in mice. Nat Aging. 2021;1(1):73-86.

[5] Naghshi S, Sadeghi O, Willett WC, Esmaillzadeh A. Dietary intake of total, animal, and plant proteins and risk of all cause, cardiovascular, and cancer mortality: systematic review and dose-response meta-analysis of prospective cohort studies. BMJ. 2020;370:m2412.

[6] Qi XX, Shen P. Associations of dietary protein intake with all-cause, cardiovascular disease, and cancer mortality: A systematic review and meta-analysis of cohort studies. Nutr Metab Cardiovasc Dis. 2020;30(7):1094-105.

[7] Song M, Fung TT, Hu FB, Willett WC, Longo VD, Chan AT, et al. Association of Animal and Plant Protein Intake With All-Cause and Cause-Specific Mortality. JAMA Intern Med. 2016;176(10):1453-63.

[8] Levine ME, Suarez JA, Brandhorst S, Balasubramanian P, Cheng CW, Madia F, et al. Low protein intake is associated with a major reduction in IGF-1, cancer, and overall mortality in the 65 and younger but not older population. Cell Metab. 2014;19(3):407-17.

[9] Chen Z, Glisic M, Song M, Aliahmad HA, Zhang X, Moumdjian AC, et al. Dietary protein intake and all-cause and cause-specific mortality: results from the Rotterdam Study and a meta-analysis of prospective cohort studies. Eur J Epidemiol. 2020;35(5):411-29.

[10] Veronese N, Li Y, Manson JE, Willett WC, Fontana L, Hu FB. Combined associations of body weight and lifestyle factors with all cause and cause specific mortality in men and women: prospective cohort study. BMJ. 2016;355:i5855.

[11] Riboli E, Hunt KJ, Slimani N, Ferrari P, Norat T, Fahey M, et al. European Prospective Investigation into Cancer and Nutrition [EPIC]: study populations and data collection. Public Health Nutr. 2002;5[6B]:1113-24.

[12] Palli D, Berrino F, Vineis P, Tumino R, Panico S, Masala $\mathrm{G}$, et al. A molecular epidemiology project on diet and cancer: the EPIC-Italy Prospective Study. Design and baseline characteristics of participants. Tumori. 2003;89(6): 586-93.

[13] Pala V, Sieri S, Palli D, Salvini S, Berrino F, Bellegotti M et al. Diet in the Italian EPIC cohorts: presentation of data and methodological issues. Tumori. 2003;89[6]:594-607.

[14] Salvini S, Parpinel M, Gnagnarella P, Maisonneuve P, Turrini A. Food Composition Database for Epidemiological Studies in Italy. 1998. (http://www.bda-ieo.it/wordpress/en/). 1998.

[15] Agnoli C, Krogh V, Grioni S, Sieri S, Palli D, Masala G et al. A priori-defined dietary patterns are associated with reduced risk of stroke in a large Italian cohort. J Nutr. 2011;141(8):1552-8.

[16] Ainsworth BE, Haskell WL, Whitt MC, Irwin ML, Swartz AM, Strath SJ et al. Compendium of physical activities: an update of activity codes and MET intensities. Med Sci Sports Exerc. 2000;32(9 Suppl):S498-S504.

[17] Agnoli C, Grioni S, Sieri S, Sacerdote C, Ricceri F, Tumino $\mathrm{R}$ et al. Metabolic syndrome and breast cancer risk: a casecohort study nested in a multicentre italian cohort. PLoS One. 2015;10(6):e0128891.

[18] Matthews DR, Hosker JP, Rudenski AS, Naylor BA, Treacher DF, Turner RC. Homeostasis model assessment: insulin resistance and beta-cell function from fasting plasma glucose and insulin concentrations in man. Diabetologia. 1985;28(7):412-9.

[19] Deutz NE, Bauer JM, Barazzoni R, Biolo G, Boirie Y, Bosy-Westphal A et al. Protein intake and exercise for optimal muscle function with aging: recommendations from the ESPEN Expert Group. Clin Nutr. 2014;33(6):929-36.

[20] Bauer J, Biolo G, Cederholm T, Cesari M, Cruz-Jentoft AJ, Morley JE, et al. Evidence-based recommendations for optimal dietary protein intake in older people: a position paper from the PROT-AGE Study Group. J Am Med Dir Assoc. 2013;14(8):542-59.

[21] Drummen M, Tischmann L, Gatta-Cherifi B, Adam T, Westerterp-Plantenga M. Dietary Protein and Energy Balance in Relation to Obesity and Co-morbidities. Front Endocrinol [Lausanne]. 2018;9:443.

[22] Wilson B. Protein mania: the rich world's new diet obsession. The Guardian. 2019.

[23] Mittendorfer B, Klein S, Fontana L. A word of caution against excessive protein intake. Nat Rev Endocrinol. 2020;16(1): 59-66.

[24] Rajendram R, Preedy V, Patel V. Branched Chain Amino Acids in Clinical Nutrition. 2015; Humana Press: Totowa, NJ, USA, 2015; Volume 1.

[25] Green CL, Lamming DW, Fontana L. Molecular mechanisms of dietary restriction promoting health and longevity. Nat Rev Mol Cell Biol. 2022;23(1):56-73. 
[26] McCarty MF. Vegan proteins may reduce risk of cancer, obesity, and cardiovascular disease by promoting increased glucagon activity. Med Hypotheses. 1999;53(6):459-85.

[27] Habegger KM, Heppner KM, Geary N, Bartness TJ, DiMarchi R, Tschop MH. The metabolic actions of glucagon revisited. Nat Rev Endocrinol. 2010;6(12):689-97.

[28] Dong Z, Gao X, Chinchilli VM, Sinha R, Muscat J, Winkels RM et al. Association of sulfur amino acid consumption with cardiometabolic risk factors: Cross-sectional findings from NHANES III. EClinicalMedicine. 2020;19.

[29] Laeger T, Henagan TM, Albarado DC, Redman LM, Bray GA, Noland RC, et al. FGF21 is an endocrine signal of protein restriction. J Clin Invest. 2014;124(9):3913-22.

[30] Huang J, Liao LM, Weinstein SJ, Sinha R, Graubard BI, Albanes D. Association Between Plant and Animal Protein Intake and Overall and Cause-Specific Mortality. JAMA Intern Med. 2020;180(9):1-12.

[31] Sun Y, Liu B, Snetselaar LG, Wallace RB, Shadyab $\mathrm{AH}$, Kroenke $\mathrm{CH}$, et al. Association of Major Dietary Protein Sources With All-Cause and Cause-Specific Mortality: Prospective Cohort Study. J Am Heart Assoc. 2021; 10(5): $\mathrm{e} 015553$.

[32] Budhathoki S, Sawada N, Iwasaki M, Yamaji T, Goto A, Kotemori A et al. Association of Animal and Plant Protein Intake With All-Cause and Cause-Specific Mortality in a Japanese Cohort. JAMA Intern Med. 2019;179(11):1509-18.

[33] Vernon ST, Coffey S, Bhindi R, Soo Hoo SY, Nelson GI, Ward $\mathrm{MR}$ et al. Increasing proportion of ST elevation myocardial infarction patients with coronary atherosclerosis poorly explained by standard modifiable risk factors. Eur J Prev Cardiol. 2017;24(17):1824-30.

[34] Lloyd-Jones DM, Leip EP, Larson MG, D’Agostino RB, Beiser A, Wilson PW et al. Prediction of lifetime risk for cardiovascular disease by risk factor burden at 50 years of age. Circulation. 2006;113(6):791-8.
[35] Joosten MM, Pai JK, Bertoia ML, Rimm EB, Spiegelman D, Mittleman MA, et al. Associations between conventional cardiovascular risk factors and risk of peripheral artery disease in men. JAMA. 2012;308(16):1660-7.

[36] Smith GI, Commean PK, Reeds DN, Klein S, Mittendorfer B. Effect of Protein Supplementation During Diet-Induced Weight Loss on Muscle Mass and Strength: A Randomized Controlled Study. Obesity (Silver Spring). 2018;26(5): 854-61.

[37] Whitehead A, Beck EJ, Tosh S, Wolever TM. Cholesterollowering effects of oat B-glucan: a meta-analysis of randomized controlled trials. Am J Clin Nutr. 2014;100(6):1413-21.

[38] Yang Y, Zhao LG, Wu QJ, Ma X, Xiang YB. Association between dietary fiber and lower risk of all-cause mortality: a meta-analysis of cohort studies. Am J Epidemiol. 2015;181(2):83-91.

[39] Siri-Tarino PW, Sun Q, Hu FB, Krauss RM. Saturated fat, carbohydrate, and cardiovascular disease. Am J Clin Nutr. 2010;91(3):502-9.

[40] Liu S, Manson JE, Buring JE, Stampfer MJ, Willett WC, Ridker PM. Relation between a diet with a high glycemic load and plasma concentrations of high-sensitivity C-reactive protein in middle-aged women. Am J Clin Nutr. 2002;75(3):492-8.

[41] Pereira MA, Swain J, Goldfine AB, Rifai N, Ludwig DS. Effects of a low-glycemic load diet on resting energy expenditure and heart disease risk factors during weight loss. JAMA. 2004;292(20):2482-90.

[42] Ferdowsian HR, Barnard ND. Effects of plant-based diets on plasma lipids. Am J Cardiol. 2009;104(7):947-56. 